\title{
Non-Unity Stoichiometric Reversible Electrode Reactions. The Effect of Coupled Kinetics and the Oxidation of Bromide
}

\author{
Haotian Chen" ${ }^{1}$, Archana Kaliyaraj Selva Kumar ${ }^{1}$, Haonan Le, Richard G. Compton* \\ Department of Chemistry, Physical and Theoretical Chemistry Laboratory, Oxford University, South Parks \\ Road, Oxford OX1 3QZ, United Kingdom
}

\section{Keywords}

Electrode reaction mechanism; Non-unity reaction stoichiometry; Bromine/bromide electrode reaction; Electrochemical tribromide dissociation; EC reaction.

\section{Abstract}

We explore via simulation, the cyclic voltammetry of the following mechanism involving a reversible electrode process coupled with a follow up reaction in which the product $(B)$ of the electrode reaction reacts chemically with the reactant, $A$, to form a species $C$ :

$$
\begin{gathered}
2 A-2 e^{-} \rightleftarrows B \\
B+A \rightleftharpoons_{k_{b}}^{k_{f}} C
\end{gathered}
$$

Such a mechanism is shown, under certain critical combinations of rate constant and reactant concentration to give rise to two voltammetric peaks despite the assumption of a single redox couple $(A / B)$. This results from the formation of $C$ during the first peak whilst the second peak, seen at more oxidizing potentials, reflects the dissociation of $C$ releasing $A$. A mechanism of the type investigated may hold during the oxidation of bromide $\left(B r^{-}, A\right)$ in solution to form bromine $\left(B r_{2}, B\right)$ and tribromide $\left(B r_{3}^{-}, C\right)$. Simulations are used to explore the conditions for which two peaks are expected in the bromide oxidation system in aqueous solution and for which the two following limiting cases operate

$$
3 \mathrm{Br}^{-}-2 e^{-} \rightleftarrows B r_{3}^{-}
$$

* Corresponding author.

Email address: $\underline{\text { Richard.compton@chem.ox.ac.uk (R. G. Compton) }}$

1. These authors contribute equally to this work. 
and

$$
2 \mathrm{Br}^{-}-2 e^{-} \rightleftarrows B r_{2}
$$

We report experimental data for the bromide oxidation in aqueous solution. These are consistent with simulations based on independent literature values for the rate and equilibrium constants for tribromide formation.

\section{Introduction}

Cyclic voltammetry at macro-electrodes is the technique at the heart of modern electrochemistry yielding qualitative important information such as electrode reaction mechanisms and pathways together with quantitative data such as equilibrium, rate and adsorption constants, and diffusion coefficients. The characterisation of electrochemically reversible voltammograms in terms of peak currents and their voltage scan rate dependence and peak to peak separations are well established for the simple oneelectron process

$$
A-e^{-} \rightleftarrows B
$$

where the Randles-Ševčík equation describes the peak currents, $I_{p},[1,2]$

$$
I_{p}=0.446 F A[A]_{b u l k} \sqrt{\frac{F D v}{R T}}
$$

where $F, R$ and $T$ are the Faraday constant, Gas constant and absolute temperature respectively. $A$ is the area of electrode, $[A]_{b u l k}$ is the bulk concentration of reductant, $D$ is the diffusion coefficient of the reductant, and $v$ is the scan rate. Eq. (2) allows the inference of the diffusion coefficient of $A$, or, alternatively, if this is already known, can allow the inference of the overall stoichiometry as that given by Eq. (1).

Whilst Eq. (2) is very widely used to interpret voltammetry, it is very important to recognise that it applies exclusively to electrode reactions of a unity stoichiometry. Thus for the general reaction

$$
a A-n e^{-} \rightleftarrows b B
$$

Eq. (2) is applicable for the case $a=b$. However, it is not valid for the frequent case of a not equal to $\mathrm{b}$. as has recently been clarified both elegantly and definitively by Molina and colleagues [3]. Specifically Molina et al highlight the fact that the peak currents in the case of electrode reactions of non-unity stoichiometry deviate markedly from the predictions of Eq. (2) with implications for the inference of 
electrode reaction mechanisms as well as for the reliability of quantitative inferences, for example of diffusion coefficients, if the equation is mis-applied.

One particularly important electrode reaction of non-unity stoichiometry is that of the oxidation of bromide ions

$$
2 B r^{-}-2 e^{-} \rightleftarrows B r_{2}
$$

which is of importance in batteries, specifically redox flow batteries and electrochemical capacitors [4-9]. The reaction is complicated because of the following chemical reaction in which the bromine product combines reversibly with the bromide reactant to form the tribromide ion,

$$
B r_{2}+B r^{-} \rightleftarrows B r_{3}^{-}
$$

so that in the presence of a large excess of bromide the electrode process tends to the following limiting overall reaction

$$
3 B r^{-}-2 e^{-} \rightleftarrows B r_{3}^{-}
$$

The reaction is well studied in both aqueous and non-aqueous media (including acetic acid, acetonitrile, nitrobenzene and some room temperature ionic liquids [10-13]). In the case of aqueous media the equilibrium constant is reported as $17 \mathrm{M}^{-1}$ at $25^{\circ} \mathrm{C}$ [14] whilst the forward rate constant for reaction (5) is reported as $1.5 \times 10^{9} M^{-1} \mathrm{~s}^{-1}[15]$

The purpose of this paper is to explore the consequence of the follow up solution phase chemistry on the predicted voltammetry of the bromide oxidation system using both simulation and experiment. It concludes that with very special combination of rate constants and voltage scan rates, two voltammetric peaks are discernible despite the occurrence of the sole electron transfer process given in Eq. (4).

\section{Theory}

We consider the electrochemically reversible, heterogeneous, one-electron oxidation of species $A$ to $B$, followed by a homogeneous chemical reaction of $A$ with $B$, as described in the introduction, with diffusiononly mass transport at a planar macro-electrode. We assume the presence of excess supporting electrolyte to suppress migration [16] and voltammetry on a sufficiently short timescale to avoid natural convection effects $[17,18]$. The reaction scheme is: 


$$
\begin{gathered}
2 A-2 e^{-} \rightleftarrows B \\
B+A \rightleftharpoons{ }_{k_{b}}^{k_{f}} C
\end{gathered}
$$

The electrode kinetics are assumed to be described by the Butler Volmer theory of electrode kinetics [19, 20], and for the mechanism $2 A-2 e^{-} \rightleftarrows B$,

$$
j=k_{0} \exp \left[\frac{\beta F}{R T}\left(E-E_{f}^{0}\right)\right]\left(\frac{c_{A}(0, t)}{[A]^{0}}\right)^{2}-k_{0} \exp \left[-\frac{\alpha F}{R T}\left(E-E_{f}^{0}\right)\right]\left(\frac{c_{B}(0, t)}{[A]^{0}}\right)
$$

where $\mathrm{j}$ is the net flux of the oxidant on the surface of the electrode, $k_{0}$ is the standard electrochemical rate constant, $c_{A}(0, t)$ and $c_{B}(0, t)$ are the concentrations of species $A$ and $B$ on the surface of electrode at time $t$. Note that $[A]^{0}$ is a standard concentration, conventionally given the value of $1 \mathrm{M}$, but as will become apparent below, for the purposes of the simulation reported it is more convenient to define the standard state as the bulk concentration of species $\mathrm{A}$ namely $c_{A}^{*}$. The quantities $F, R$, and $T$ are the Faraday Constant, the Gas Constant and the absolute temperature respectively. $\alpha$ and $\beta$ are the transfer coefficients, where usually, but not always, for a two-electron transfer process, $\alpha+\beta=2$ at potentials close to equilibrium. In the following simulation the electrochemical rate constant, $k_{0}$, is made artificially high to ensure the process is Nernstian in character and hence independent of the values of $\alpha$ and $\beta$ subject to their summing to 2 (see SI).

Note that when the net flux is zero Eq. (8) reduces to the Nernst equation in which the standard concentration [ $]^{0}$ is defined to be $1 \mathrm{M}$, and the Nernst Equation can be written as

$$
E=E_{f}^{0}(A / B)+\frac{R T}{F} \ln \left(\frac{\left(\frac{[B]}{[]^{0}}\right)^{\frac{1}{2}}}{\frac{[A]}{[]^{0}}}\right)
$$

However, if during simulation, the standard concentration is the bulk concentration of bromide, $[A]^{0}$, it is helpful to define a new formal potential should be derived and a new factor $\mathrm{q}$ is introduced as:

$$
q=\frac{[A]^{0}}{[]^{0}}
$$


So the new formal potential, $\mathrm{E}_{f}^{0 \prime \prime}$, related to standard state $[A]^{0}$ is:

$$
\begin{aligned}
E & =E_{f}^{0^{\prime \prime}}(A / B)+\frac{R T}{F} \ln \left(\frac{\left(\frac{[B]}{[A]^{0}}\right)^{\frac{1}{2}}}{\frac{[A]}{[A]^{0}}}\right)=E_{f}^{0^{\prime \prime}}(A / B)+\frac{R T}{F} \ln \left(\frac{\left(\frac{[B]}{q[]^{0}}\right)^{\frac{1}{2}}}{\frac{[A]}{q[]^{0}}}\right) \\
& =E_{f}^{0^{\prime \prime}}(A / B)+\frac{R T}{F} \ln \left(\frac{\left(\frac{[B]}{[]^{0}}\right)^{\frac{1}{2}}}{\frac{[A]}{[]^{0}}}\right)+\frac{R T}{F} \ln \left(q^{\frac{1}{2}}\right)
\end{aligned}
$$

And,

$$
E_{f}^{0}(A / B)=E_{f}^{0^{\prime \prime}}(A / B)+\frac{R T}{F} \ln \left(q^{\frac{1}{2}}\right)
$$

Hence the new formal potential of A/B couple should be:

$$
E_{f}^{0^{\prime \prime}}(A / B)=E_{f}^{0}(A / B)-\frac{R T}{F} \ln \left(q^{\frac{1}{2}}\right)
$$

\section{Reaction Model}

The heterogeneous electrochemical reaction followed by homogeneous chemical reaction is simulated on a planar macroelectrode. The scale of the electrode is assumed to be significantly larger than the distance the species can diffuse during the timescale of simulation, hence the model is simplified to a onedimensional system, and only diffusion perpendicular to the electrode surface is taken into consideration. Using Fick's second law of diffusion [21, 22], the diffusion equation can be expressed as:

$$
\frac{\partial c_{j}}{\partial t}=D_{j} \nabla^{2} c_{j}=D \frac{\partial^{2} c_{j}}{\partial x^{2}}
$$


where $j$ is the species under study, and $D_{j}$ is the diffusion coefficient.

The variation in concentration of species in the solution phase can be simulated by considering Fick's second law of diffusion with second order homogeneous chemical reaction, when one $A$ molecule and one $\mathrm{B}$ molecule react to form $\mathrm{C}$ reversibly. The process of the forward reaction, $\mathrm{A}$ and $\mathrm{B}$ forming $\mathrm{C}$, is controlled by the rate constant $k_{f}$ and the backward reaction is controlled by rate constant $k_{b}$.

$$
\left\{\begin{array}{l}
\frac{\partial c_{A}}{\partial t}=D_{A} \frac{\partial^{2} c_{A}}{\partial x^{2}}-k_{f} c_{A} c_{B}+k_{b} c_{C} \\
\frac{\partial c_{B}}{\partial t}=D_{B} \frac{\partial^{2} c_{B}}{\partial x^{2}}-k_{f} c_{A} c_{B}+k_{b} c_{C} \\
\frac{\partial c_{C}}{\partial t}=D_{A} \frac{\partial^{2} c_{C}}{\partial x^{2}}+k_{f} c_{A} c_{B}-k_{b} c_{C}
\end{array}\right.
$$

To solve the differential equations, both temporal and spatial boundary conditions need to be specified. At the beginning of simulation, the bulk concentration of species $A$ is set to $c_{A}^{*}$, with no $B$ or $C$ present in the system.

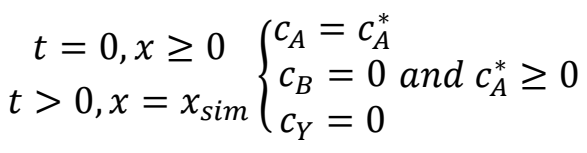

where $\mathrm{x}_{\text {sim }}$ is the outer boundary for simulation, distant enough from the electrode that the concentration of each species equal to its bulk concentration. Thus, the boundary of simulation needs to be sufficiently far from the electrode that the reaction on the surface of the electrode would not affect the condition on the outer boundary. From Einstein's work on Brownian motion [23], in a one-dimensional system for a particle, $\sqrt{\left\langle x^{2}\right\rangle}=\sqrt{2 D t_{\text {sim }}}$, which can be used to approximate the distance from the electrode that is unperturbed from the reaction on the electrode surface. So, the distance of outer boundary for simulation is chosen to be greater or equal to this:

$$
x_{\text {sim }}=6 \sqrt{D t_{\text {sim }}}
$$

The boundary conditions at the surface of the electrode reflects the electrochemical activity of the species in the potential range of study [24]: 


$$
\left\{\begin{array}{l}
D_{A}\left(\frac{\partial c_{A}}{\partial x}\right)_{x=0}=k_{0} \exp \left[\frac{\beta F}{R T}\left(E-E_{f}^{0}\right)\right]\left(\frac{c_{A}(0, t)}{[A]^{0}}\right)^{2}-k_{0} \exp \left[-\frac{\alpha F}{R T}\left(E-E_{f}^{0}\right)\right]\left(\frac{c_{B}(0, t)}{[A]^{0}}\right) \\
D_{B}\left(\frac{\partial c_{B}}{\partial_{x}}\right)_{x=0}=-\frac{1}{2} D_{A}\left(\frac{\partial c_{A}}{\partial x}\right)_{x=0} \\
D_{C}\left(\frac{\partial c_{C}}{\partial x}\right)_{x=0}=0
\end{array}\right.
$$

where $A$ and $B$ are electro-active species and follow Butler-Volmer kinetics, whist $C$ is electrochemical inactive at the potential range under study, and thus has zero-flux at the electrode surface.

\section{Dimensionless Parameters}

Dimensionless parameters, as defined in Table 1, are used for simulation instead of dimensional ones. Dimensionless parameters remove the dependence on bulk concentration, $c_{A}^{*}$, radius of electrode, $\epsilon$, diffusion coefficient, $D$ and formal potentials, $E_{f}^{0}$; and the resulting voltammograms can be applied to any set of values of $c_{A}^{*}, \epsilon, D$, and $E_{f}^{0}$. The simulation is therefore both more significant and general. The dimensionless parameters are generally obtained by multiplying the dimensional parameters with a certain set characteristic value.

\section{Table 1}

List of definitions of dimensionless parameters for planar electrode simulations of the system interest.. $c_{j}$ is the concentration of species $j ; c_{A}^{*}$ and $D_{A}$ are the bulk concentration of species $A . \epsilon$ is the radius of planar electrode, assumed to be the flat surface of a circular disc. $E_{f}^{0 \prime \prime}$ is the formal potential used for simulation at standard state equal to bulk concentration of $A, c_{A}^{*}$.

\section{Dimensionless Parameter}

Concentration of species j

Diffusion coefficient of species $j$

Spatial Coordinates

Potential

Scan Rate

\section{Definition}

$$
\begin{gathered}
C_{j}=\frac{c_{j}}{c_{A}} \\
d_{j}=\frac{D_{j}}{D_{A}} \\
X_{i}=\frac{x_{i}}{\epsilon} \\
\theta=\frac{F}{R T}\left(E-E_{f}^{0 \prime \prime}\right) \\
\sigma=\frac{\epsilon^{2}}{D_{A}} \frac{F}{R T} v
\end{gathered}
$$


Current

$$
J=\frac{I}{\pi \epsilon F D_{A} c_{A}^{*}}
$$

Standard Electrochemical Rate Constant

$$
K_{0}=\frac{k_{0} \epsilon}{D_{A} c_{A}^{*}}
$$

Chemical Rate Constant, First Order

$$
K_{b}=\frac{k_{b} \epsilon^{2}}{D_{A}}
$$

Chemical Rate Constant, Second Order

$$
K_{f}=\frac{k_{f} c_{A}^{*} \epsilon^{2}}{D_{A}}
$$

The dimensionless diffusion equation can be written as:

$$
\left\{\begin{array}{l}
\frac{\partial C_{A}}{\partial T}=d_{A} \frac{\partial^{2} C_{A}}{\partial X^{2}}-K_{f} C_{A} C_{B}+K_{b} C_{C} \\
\frac{\partial C_{B}}{\partial T}=d_{B} \frac{\partial^{2} C_{B}}{\partial X^{2}}-K_{f} C_{A} C_{B}+K_{b} C_{C} \\
\frac{\partial C_{C}}{\partial T}=d_{A} \frac{\partial^{2} C_{C}}{\partial X^{2}}+K_{f} C_{A} C_{B}-K_{b} C_{C}
\end{array}\right.
$$

The boundary condition at the start of the simulation and at the surface of the electrode can be written in dimensionless form as:

$$
\begin{gathered}
T=0, X \geq 0 \quad\left\{\begin{array}{c}
C_{A}=1 \\
C_{B}=0 \\
C_{Y}=0
\end{array}\right. \\
\left\{\left(\frac{\partial C_{A}}{\partial X}\right)_{X=0}=K_{0} \exp (\beta \theta)\left(C_{A}(0, T)\right)^{2}-k_{0} \exp (-\alpha \theta) C_{B}(0, T)\right. \\
d_{B}\left(\frac{\partial C_{B}}{\partial X}\right)_{X=0}=-\frac{1}{2}\left(\frac{\partial C_{A}}{\partial X}\right)_{X=0} \\
d_{C}\left(\frac{\partial C_{C}}{\partial X}\right)_{X=0}=0
\end{gathered}
$$




\section{Simulation Methods}

Simulation was performed using a home written C++ programs with std::thread for parallel computing running on an Intel Core i7 CPU. The differential equations were solved using the Newton-Raphson method [17] with at most eight iterations required for convergence. Graphs were plotted with Origin Pro 2019 and Python Matplotlib. Convergence studies were performed to and evaluated using various checks involving the magnitude of the peak currents and potentials and checking mass conservation[25-27]. The value of peak current and peak to peak separation matched with the values predicted by Molina's work [3]. Details of mass conservation and convergence studies are presented in the SI.

\section{Theoretical Results}

First simulations were made of a simple fully reversible $2 A-2 e^{-} \rightleftarrows B$ process without any follow-up chemical reaction to permit comparison with the published work of Molina [3]. In particular, the simulation results were in quantitative agreement with Molina's work. In particular, Molina predicted that for such a process, the peak flux $J$ in dimensionless form should be $J=0.4995 \sqrt{\sigma}$ and the peak to peak separation should be $42 \mathrm{mV}$, and in dimensionless form $\theta_{p p}=1.6$. Fig. 1 shows our simulation of a cyclic voltammogram at $\sigma=1198$, corresponding to dimensional scan rate at $100 \mathrm{mV} / \mathrm{s}$ with dimensional parameters of $\left(\epsilon=0.8 \mathrm{~mm}, c_{A}^{*}=5 \mathrm{mM}, c_{B}^{*}=0 \mathrm{mM}, c_{C}^{*}=0 M, D_{A}=2.08 \times 10^{-9} \mathrm{~m}^{2} / \mathrm{s}\right)$. The dimensionless peak flux was found to be 17.30, very close to Molina's prediction of 17.29 [3] using the equation $J=0.4995 \sqrt{\sigma}$. Similarly, the dimensionless peak to peak separation, $\theta_{p p}$, of the simulation voltammogram is 1.63, corresponding to $41.85 \mathrm{mV}$ in dimensional form, also in excellent agreement with Molina. A similar level of accuracy was obtained for different dimensionless scan rate in the range $\sigma=$ 0.1198 to $\sigma=119800$, corresponding to dimensional scan rate from $10^{-5} \mathrm{~V} / \mathrm{s}$ to $10 \mathrm{~V} / \mathrm{s}$. 


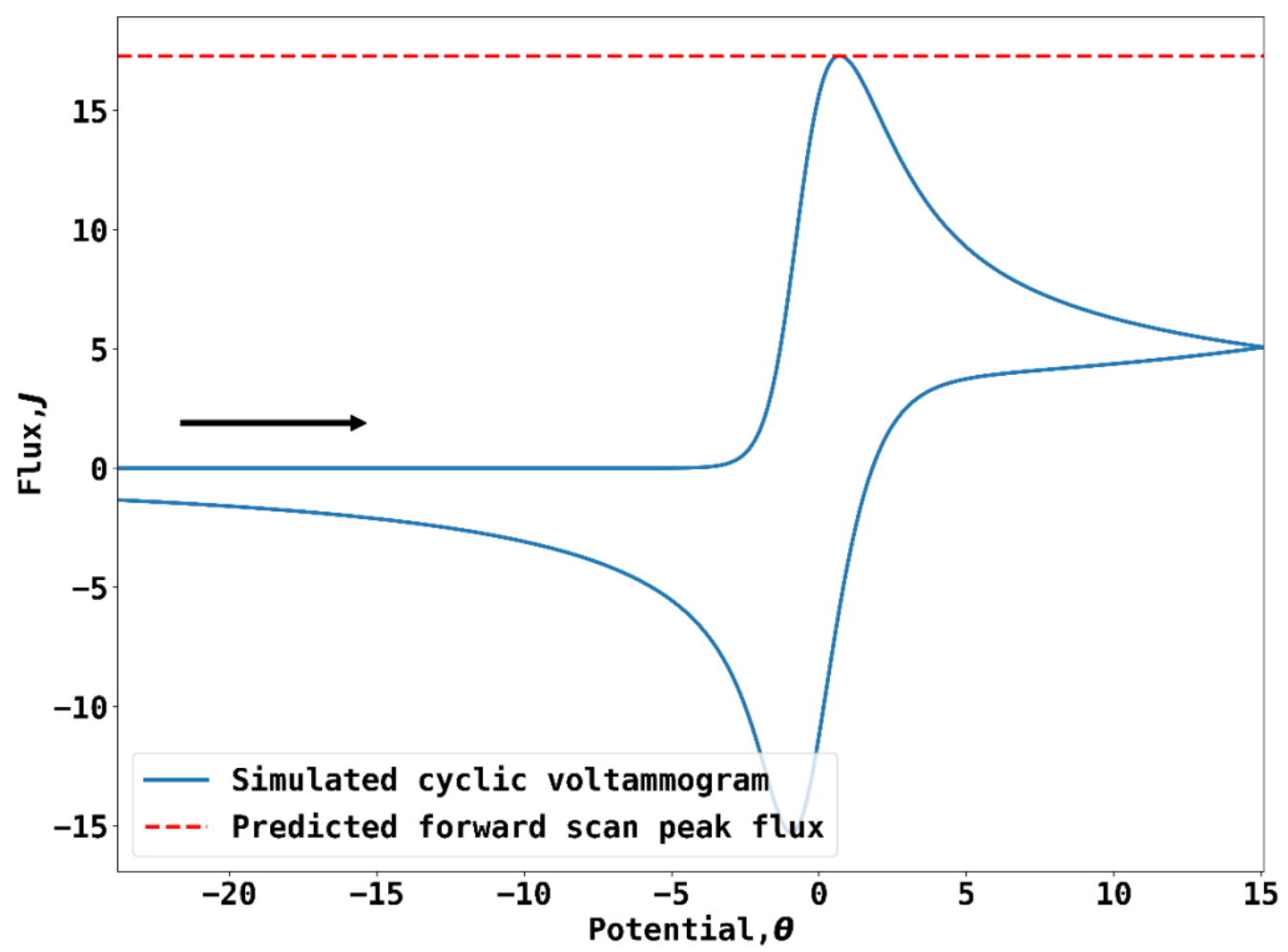

Fig. 1. Cyclic voltammogram of a fast(reversible) electrochemical reaction at $\sigma=1198$ of stoichiometry of 2:1, corresponding to $100 \mathrm{mV} / \mathrm{s}$. The dashed red line is the predicted forward scan peak flux from Molina [3]. The forward scan peak flux is 17.30 and peak to peak separation, $\theta_{p p}$ is 1.63. The black arrow indicates the start and initial direction of potential sweep.

Next we performed simulations on the two-electron oxidation process followed by homogeneous $2 A-2 e^{-} \rightleftarrows B$

chemical reaction: $B+A \underset{k_{b}}{k_{f}} C$.

Simulations were carried out on a range of parameters considering both reversible and irreversible electron transfer kinetics and varying degrees of the chemical reversibility of the chemical reaction. First, we consider reversible electron transfer.

With the follow up chemical reaction, a second peak can be observed in the voltammogram under certain conditions even though only a single electron transfer event is modeled! To observe the second peak, some important conditions should be satisfied, as is next discussed.

First, the second peak can only be seen if $k_{f}>0$ and $k_{b}>0$. In contrast if the chemical reaction is fully irreversible, i.e., $k_{f}>0$ and $k_{b}=0$, the second peak cannot be observed. A simulation of a typical electrochemically reversible cyclic voltammetry at scan rate of $\sigma=1198$, or $100 \mathrm{mV} / \mathrm{s}$ and a forward reaction rate constant, $K_{f}=1.53 \times 10^{9}$, corresponding to $k_{f}=10^{9} \mathrm{M}^{-1} \mathrm{~S}^{-1}$ with other dimensional 
parameters $\left(\epsilon=0.8 \mathrm{~mm}, c_{A}^{*}=5 \mathrm{mM}, c_{B}^{*}=0 \mathrm{mM}, c_{C}^{*}=0 M, D_{A}=2.08 \times 10^{-9} \mathrm{~m}^{2} / \mathrm{s}\right)$ develops this hypothesis. As shown in Fig. 2, for a reverse reaction rate constant, $K_{b}=3.08 \times 10^{7}$, corresponding to dimensional form, $k_{b}=10^{5} \mathrm{~s}^{-1}$ and if the chemical reaction is reversible, the second peak can be observed. But when $K_{b}=0$, or $k_{b}=0 s^{-1}$, the second peak no longer appears. This behavior suggests that the presence of the second peak can only be seen when the chemical reaction is reversible, and is related to the backward chemical reaction, the decomposition of $C$ to form $A$ and $B$.

Since we ascribe the second peak to the dissociation of $C$ formed during the oxidation of $A$ to $B$ on the voltammetric timescale, next simulations were made to explore more fully the conditions under which a second peak was visible.

Although the chemical reaction is required to be reversible to see the second peak, the electrochemical reaction can be either reversible (as above) or irreversible. For example, simulations were made when $K_{0}=0.1$, small enough to ensure irreversibility, with the dimensionless chemical reaction rate constants are $K_{f}=1.53 \times 10^{9}$ and $K_{b}=3.08 \times 10^{7}$ respectively, corresponding to $k_{f}=10^{9} M^{-1} s^{-1}$ and $k_{b}=$ $10^{5} \mathrm{~s}^{-1}$ with other dimensional parameters $\left(\epsilon=0.8 \mathrm{~mm}, c_{A}^{*}=5 \mathrm{mM}, c_{B}^{*}=0 \mathrm{mM}, c_{C}^{*}=0 \mathrm{M}, D_{A}=\right.$ $\left.2.08 \times 10^{-9} \mathrm{~m}^{2} / \mathrm{s}\right)$. In Fig. 3, the resulting voltammograms shows that for irreversible electrode kinetics, the second peak can still be observed at around $\theta=14$.

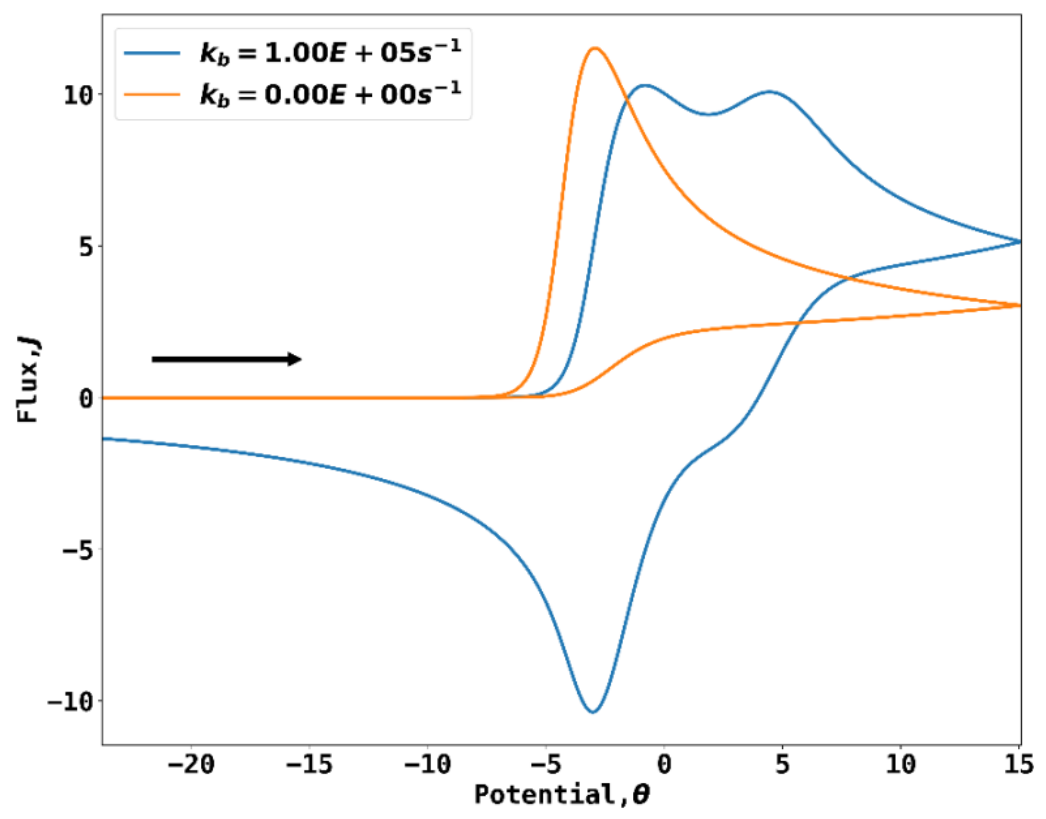

Fig. 2. Simulated voltammetry showing that when $k_{f}=10^{9} \mathrm{M}^{-1} \mathrm{~S}^{-1}$ and $k_{b}=10^{5} \mathrm{~s}^{-1}$, the second peak can observed(blue line). But when $k_{f}=10^{9} \mathrm{M}^{-1} \mathrm{~s}^{-1}$ and $k_{b}=0$, the second peak cannot be observed (orange line). The dimensionless electrochemical rate constant is set to be $K_{0}=10^{9}$ in both cases. The black arrow indicates the start and initial direction of potential sweep. 


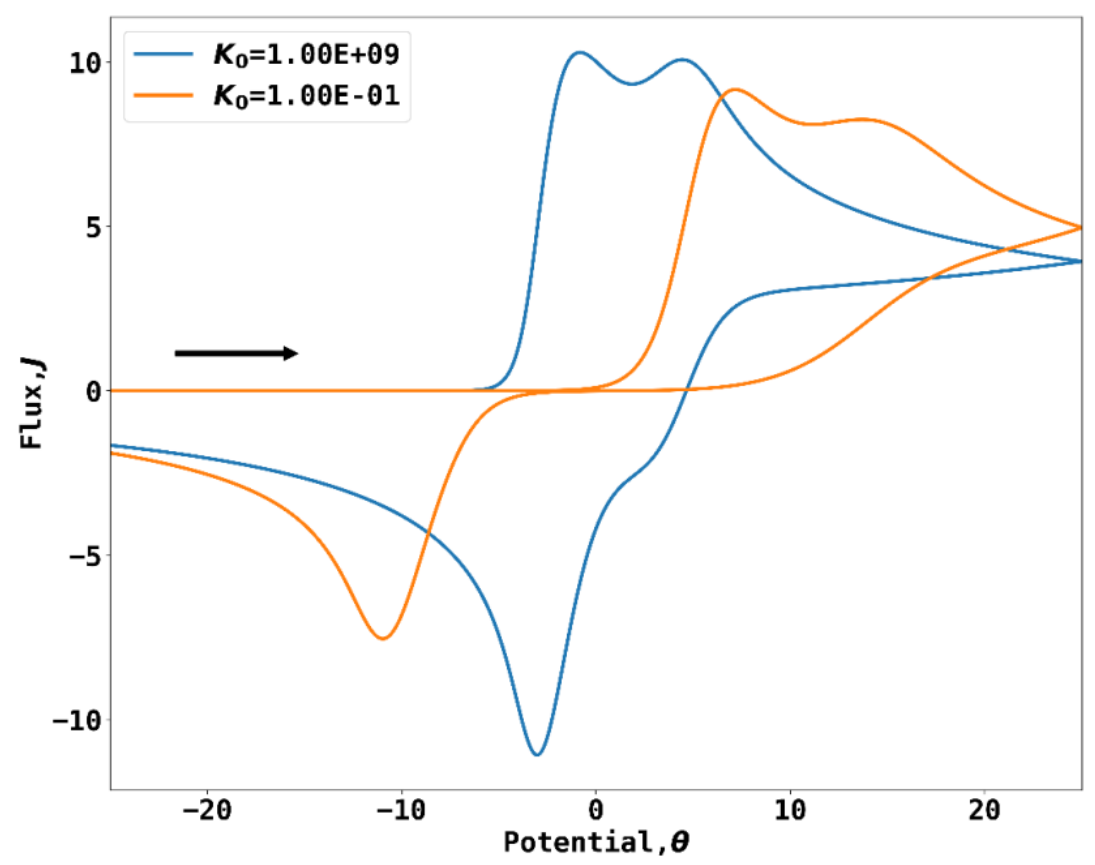

Fig. 3. Simulated voltammetry showing that when $k_{f}=10^{9} M^{-1} S^{-1}$ and $k_{b}=10^{5} \mathrm{~s}^{-1}$, the electrochemical rate constant is varied from $K_{0}=10^{9}$ to $K_{0}=10^{-1}$ a second peaks can be observed in both cyclic voltammograms with fully reversible electrode kinetics $\left(K_{0}=10^{9}\right)$ and fully irreversible electrode kinetics $\left(K_{0}=10^{-1}\right)$.

Second simulations were conducted to see the influence of the bulk concentration of $A$ on the appearance or otherwise of the second peak. Simulation was performed at different $c_{A}^{*}$ from $0.1 \mathrm{M}$ to $10 \mathrm{M}$ with the following dimensional parameters $\left(\epsilon=0.8 \mathrm{~mm}, c_{B}^{*}=0 \mathrm{mM}, c_{C}^{*}=0 M, D_{A}=2.08 \times 10^{-9} \mathrm{~m}^{2} / \mathrm{s}\right)$ at a scan rate $\sigma=1198$, or $v=0.1 \mathrm{~V} / \mathrm{s}$. The chemical rate constant modelled was that $K_{f}=1.53 \times 10^{9}$, corresponding to $k_{f}=10^{9} M^{-1} s^{-1}$ and $K_{e q}=500$,or $k_{e q}=1 e^{5} M^{-1}$. As shown in Fig. 4 , the second peak appears at lower bulk concentration at $c_{A}^{*}=0.1 \mathrm{M}$ around $\theta=9$ and disappears as $c_{A}^{*}$ increases to $1 \mathrm{M}$ and above. From the simulations above can conclude that the presence of the second peak also depends on the bulk concentration of species, and usually appears when the bulk concentration of $A$ is low so facilitating the dissociation of $C$. 


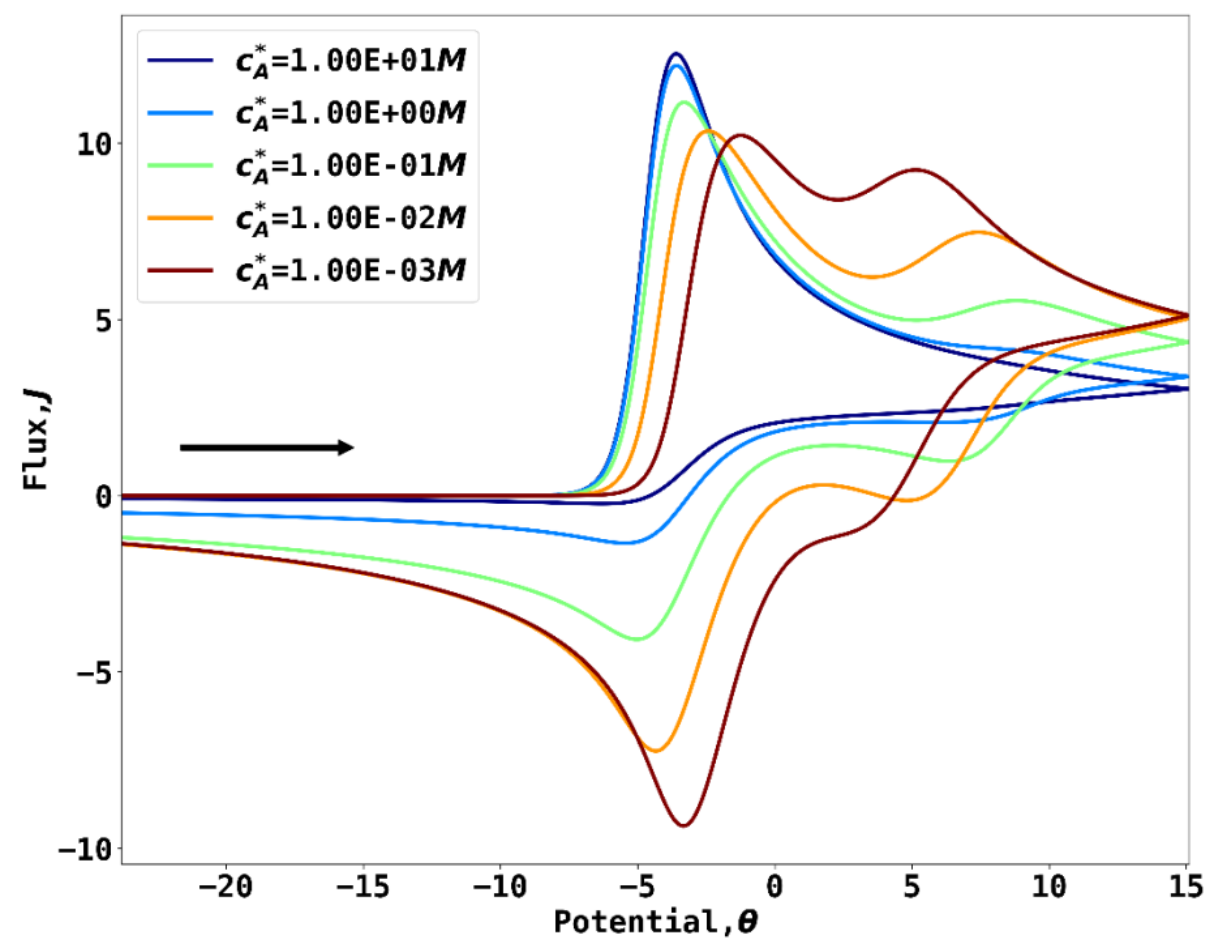

Fig. 4. Simulations performed at different $c_{A}^{*}$ from $0.1 \mathrm{M}$ to $10 \mathrm{M}$ when $k_{e q}$ is $10^{5} \mathrm{M}^{-1}$. From the figure can be observed that the second peak appears at $\theta=9$ when $c_{A}^{*}=0.1 \mathrm{M}$ and disappears at higher bulk concentration. The black arrow indicates the start and initial direction of potential sweep.

Third, simulations were made to show that the peak can first appear and then disappear as the scan rate is changed. The scan rates analyzed were from $\sigma=0.12$ to $\sigma=1.2 \times 10^{8}$, corresponding to dimensional scan rate of $10^{-5} \mathrm{~V} / \mathrm{s}$ to $10^{3} \mathrm{~V} / \mathrm{s}$. A Standard electrochemical rate constant, $K_{0}$ is set to $10^{9}$, or in dimensional form $k_{0}=13 \mathrm{~m} / \mathrm{s}$, large enough to ensure reversibility, and the chemical reaction rate constant $K_{f}=1.53 \times 10^{9}$, or $k_{f}=1 \times 10^{9} \mathrm{M}^{-1} \mathrm{~s}^{-1}$, and $K_{e q}=500$, or $k_{e q}=10^{5} \mathrm{M}^{-1}$ in dimensional form, with the dimensional parameters $\left(\epsilon=0.8 \mathrm{~mm}, c_{A}^{*}=5 \mathrm{mM}, c_{B}^{*}=0 \mathrm{mM}, c_{C}^{*}=0 M, D_{A}=2.08 \times\right.$ $10^{-9} \mathrm{~m}^{2} / \mathrm{s}$ ). As shown in Fig. 5 , by varying scan rate from very small to large, the second peak can first be seen at lower scan rate from $\sigma=0.12$ to $\sigma=1.2 \times 10^{5}$ at dimensionless potential around $\theta=5$, but disappear when $\theta=1.2 \times 10^{6}$ and higher. Note that the flux is normalized by dividing with the square root of scan rate. 


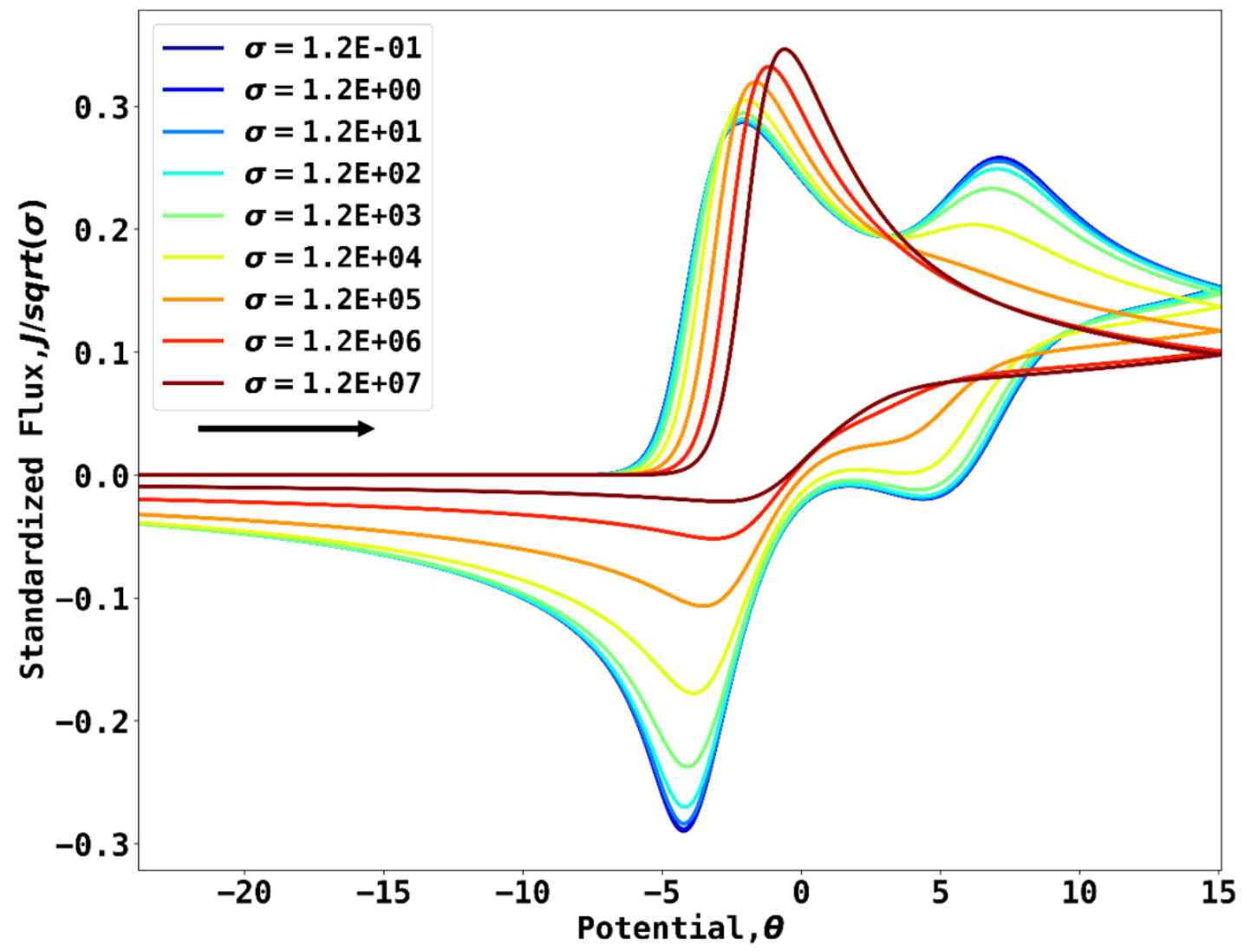

Fig. 5.Simulated voltammetry showing that when $k_{f}=10^{9} \mathrm{M}^{-1} \mathrm{~S}^{-1}$ and $k_{e q}=10^{5} \mathrm{M}^{-1}$, second peak appears at low scan rate, but disappears when the scan rate increases to $\sigma=1.2 \times 10^{7}$. The flux is normalized to the square root of scan rate. The black arrow indicates the start and initial direction of potential sweep.

Fourth simulations showed that the forward rate constant must be larger than a threshold value in order to see the second peak. During simulation, the forward reaction rate constants, $k_{f}$, were varied from $10 M^{-1} S^{-1}$ to $10^{9} M^{-1} S^{-1}$ while the equilibrium rate constant was set to $10^{5} \mathrm{M}^{-1}$ with the dimensional parameters $\left(\epsilon=0.8 \mathrm{~mm}, c_{A}^{*}=5 \mathrm{mM}, c_{B}^{*}=0 \mathrm{mM}, c_{C}^{*}=0 M, D_{A}=2.08 \times 10^{-9} \mathrm{~m}^{2} / \mathrm{s}\right)$. As shown in Fig. 6 , at the range of study, forward scan peak height decreases and the forward scan peak shifts to a more reductive potential as $k_{f}$ increases.

From $k_{f}=10^{7} M^{-1} S^{-1}$ and higher one can observe the second peak during forward scan at around $\theta=5$ and when $k_{f}$ is at $10^{9} \mathrm{M}^{-1} \mathrm{~S}^{-1}$, a second peak in the reverse scan can also be visualized. In conclusion, the second peak can be observed when the forward reaction rate constant is large enough than $10^{7} M^{-1} S^{-1}$. 


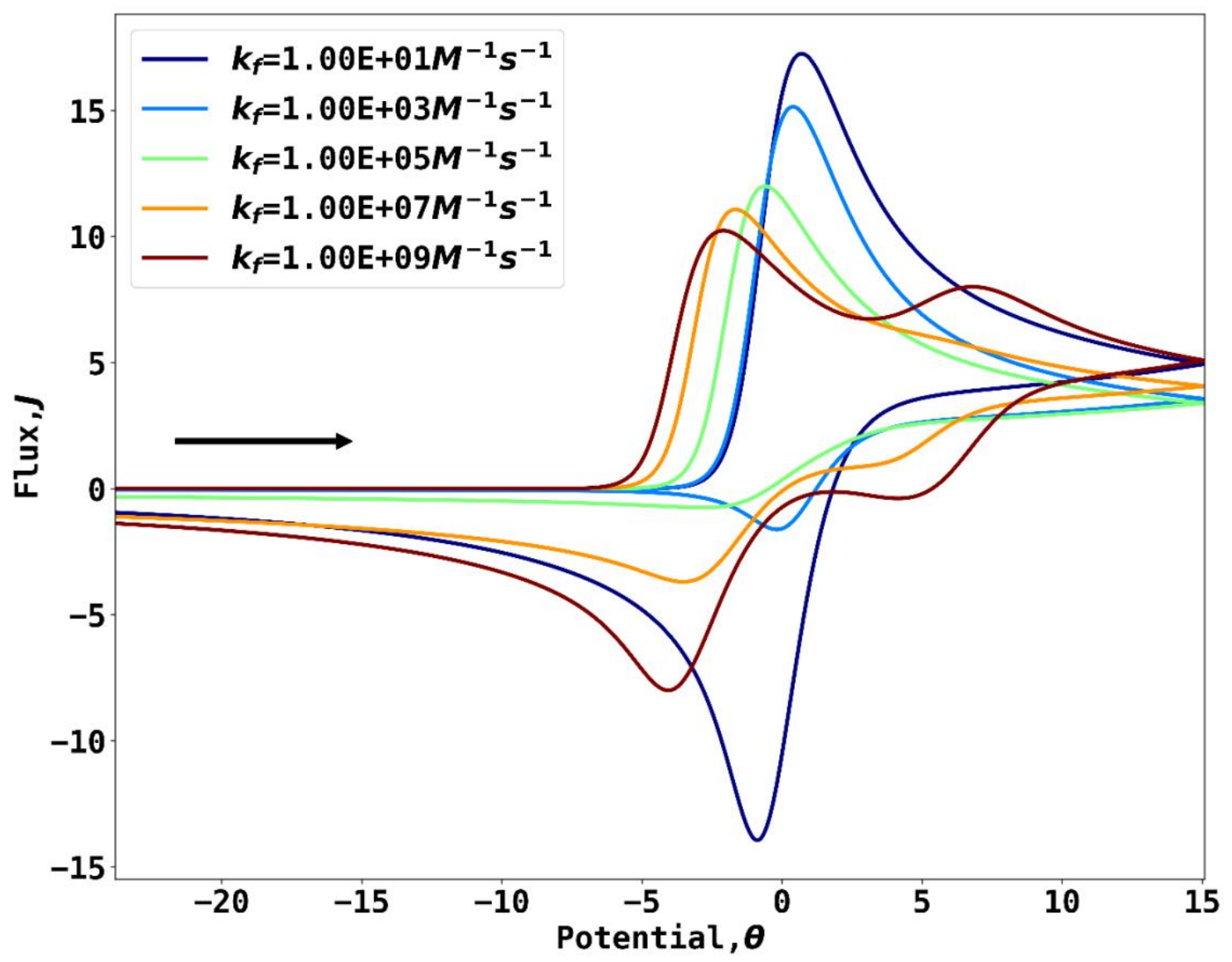

Fig. 6. Voltammetry corresponding to Forward reaction rate constant $k_{f}$ is simulated from $10 M^{-1} S^{-1}$ to $10^{9} M^{-1} S^{-1}$ with the equilibrium constant $k_{f}$ kept constant at $10^{5} \mathrm{M}^{-1}$. The black arrow indicates the start and initial direction of potential sweep.

\section{Guidance to experimentalists}

For fixed values of the forward and reverse rate constants of the following chemical reaction, it is possible to observe the second peak during experiment by altering $v$, the scan rate and $c_{A}^{*}$, the bulk concentration of species A. A series of simulations were performed to by varying $v$ from $10^{-4} \mathrm{~V} / \mathrm{s}$ to $10^{2} \mathrm{~V} / \mathrm{s}$ and $c_{A}^{*}$ from $10^{-5} M$ to $10^{2.5} M$ to show their joint influence on the presence of the second peak and its peak height. Simulations are performed with the following rate constants: $k_{f}=1 \times 10^{9} M^{-1} s^{-1}$, and $k_{e q}=$ $1 \times 10^{3} M^{-1}$ in dimensional form with the following dimensional parameters $\left(\epsilon=0.8 \mathrm{~mm}, c_{A}^{*}=\right.$ $\left.5 m M, c_{B}^{*}=0 m M, c_{C}^{*}=0 M, D_{A}=2.08 \times 10^{-9} \mathrm{~m}^{2} / \mathrm{s}\right)$. Fig. 7 shows the joint influence on the presence of second peak and its height is illustrated. The $x$ axis and $y$ axis represent the bulk concentration of species $A$ and scan rate respectively. The third dimension, $J_{p, s}$ represents the peak height of the second peak in dimensionless standardized form. The peak height is standardized as $J_{p, s}=\frac{J_{p}}{\sqrt{\sigma}}$. If $J_{p, s}$ is 0 , then no second peak can be found in the range of scanning. Fig. 7 shows that the second peak tends to appear at lower scan rate as predicted above. But the influence of concentration at the second peak is more complicated. When the bulk concentration of species $A$ is higher than $10^{-1.5} \mathrm{M}$, the second peak is more visible at 
lower concentration and can reach a maximum of $J_{p, s} \approx 0.3$. But when concentration is below the threshold of $10^{-2} M$, the second peak can no longer be present. This can be explained that at small $c_{A}^{*}$, not enough $C$ species can be decomposed to form the second peak. In general, a second peak is most visible at low scan rate, and the bulk concentration of $A$ is around $0.1 \mathrm{M}$.

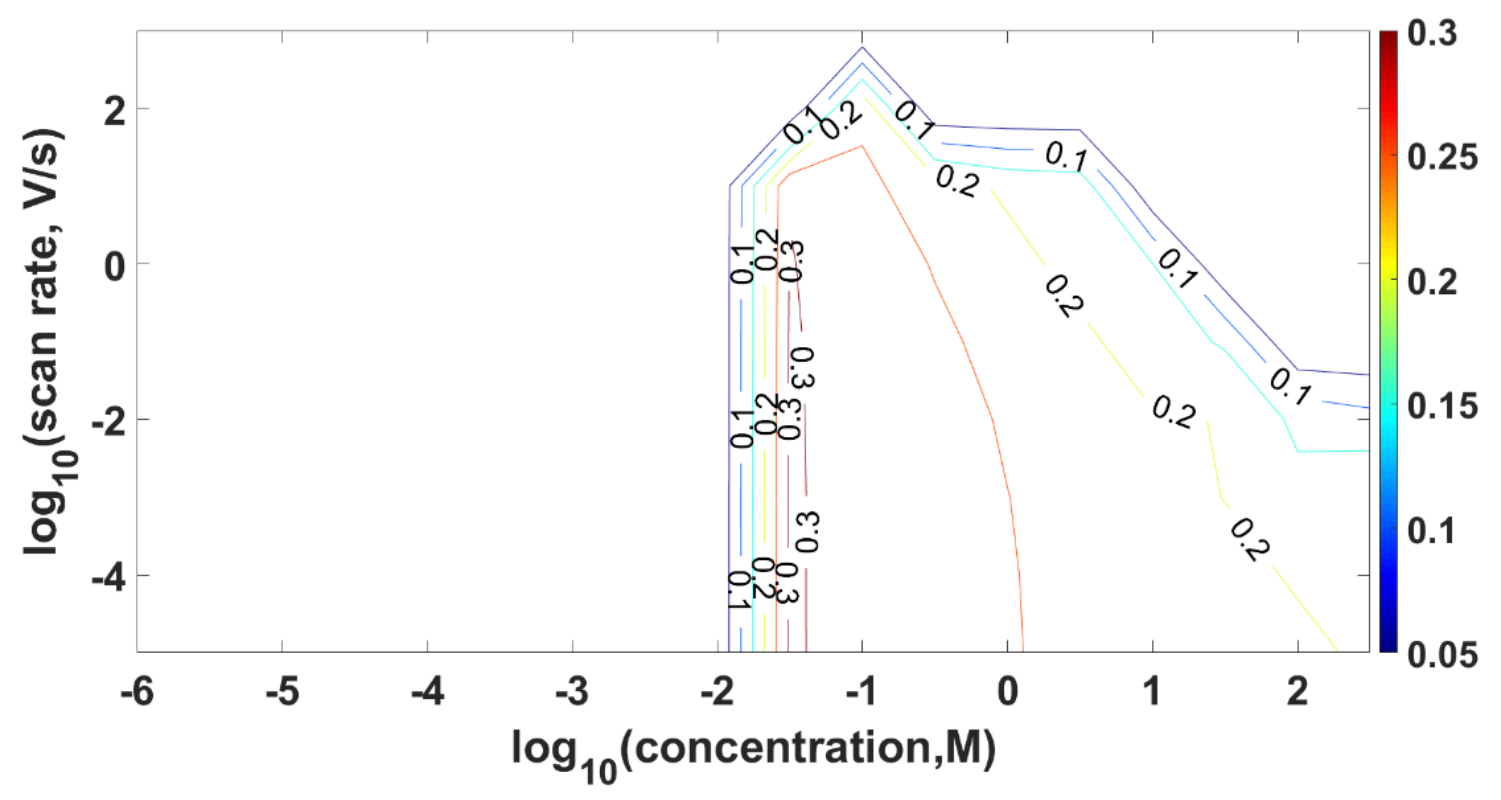

Fig. 7. Two dimensional plotting of the collective influence of bulk concentration of species $A, c_{A}^{*}$ and scan rate on the presence and height of second peak. $c_{A}^{*}$ is varied from $10^{-4} \mathrm{M}$ to $10^{2.5} \mathrm{M}$ with the scan rate varied from $10^{-4} \mathrm{~V} / \mathrm{s}$ to $10^{2} \mathrm{~V} / \mathrm{s}$. The contour plot shows the standardized peak height, and value of 0 means no second peak is observed.

\section{Simulation of the bromine/bromide system using literature kinetic data}

According to the literature[28], the electrochemical oxidation of bromide to bromine can coincide with the reversible chemical reaction of bromine and bromide to form tribromide. The mechanism is shown Eq. (22):

$$
\begin{aligned}
& 2 \mathrm{Br}^{-}-2 e^{-} \rightleftarrows \mathrm{Br}_{2} \\
& \mathrm{Br}^{-}+\mathrm{Br}_{2} \rightleftharpoons_{k_{b}}^{k_{f}} B r_{3}^{-}
\end{aligned}
$$

which fits the reaction model shown in Eq.(7). Accordingly, simulations of the bromine/bromide system in aqueous solution were made using literature rate and equilibrium constants.

The forward reaction rate constants $k_{f}$ and reverse reaction rate constant $k_{b}$ in aqueous solution are reported to be $1.5 \times 10^{9} \mathrm{M}^{-1} \mathrm{~S}^{-1}$ and $5 \times 10^{7} \mathrm{~s}^{-1}$ at $25^{\circ} \mathrm{C}$ respectively as measured using the RamanLaser Temperature-Jump technique[15]. These data are consistent with an independent measurement of 
the equilibrium constant of $17 M^{-1}$ [15]. The measured diffusion coefficients of bromide and tribromide are reported with considerable precision and to be $2.08 \times 10^{-9} \mathrm{~m}^{2} / \mathrm{s}$ and $1.145 \times 10^{-9} \mathrm{~m}^{2} / \mathrm{s}$ respectively at $25^{\circ} \mathrm{C}[29,30]$.

The diffusion coefficient of bromide was confirmed by experiments shown later in this paper. The diffusion coefficient of bromine is reported to be $1.18 \times 10^{-9} \mathrm{~m}^{2} / \mathrm{s}$ at $25^{\circ} \mathrm{C}$ [31]. The various dimensional parameters translate into dimensionless equivalents such as $K_{f}=2.31 \times 10^{9}$ and $K_{b}=$ $1.54 \times 10^{10}$ with the dimensional parameters $\left(\epsilon=0.8 \mathrm{~mm}, c_{B r^{-}}^{*}=5 \mathrm{mM}, c_{B r_{2}}^{*}=0 \mathrm{mM}, c_{B r_{3}^{-}}^{*}=0 M\right.$, $\left.D_{B r^{-}}=2.08 \times 10^{-9} \mathrm{~m}^{2} / \mathrm{s}, D_{B r_{2}}=1.18 \times 10^{-9} \mathrm{~m}^{2} / \mathrm{s}, D_{B r_{3}^{-}}=1.145 \times 10^{-9} \mathrm{~m}^{2} / \mathrm{s}\right)$. At $\sigma=1198$, corresponding to $0.1 \mathrm{~V} / \mathrm{s}$, the result of simulation is shown at Fig. 8. No second peak can be observed in the voltammogram consistent with minimal generation of tribromide. The voltammogram shows that the forward scan peak flux is only very slightly lower (ca 2\%) than Molina's prediction (red dashed line) for the bromide to bromine oxidation discounting the follow up chemical reaction. The slightly decreased peak flux can be attributed to a very small amount of competing chemical reaction of forming tribromide from bromine and bromide. The well-known Randles-Ševčík equation [1, 2] for the process $A-e \rightleftarrows B$ predicts a peak flux (green dashed line), almost $10 \%$ lower than the simulation peak flux, suggesting that if the ordinary Randles-Ševčík equation derived for unity stoichiometry reaction used to analyse experimental data then this may lead to significant errors in inferred parameters such as concentrations or diffusion coefficients. This is explored experimentally below. 


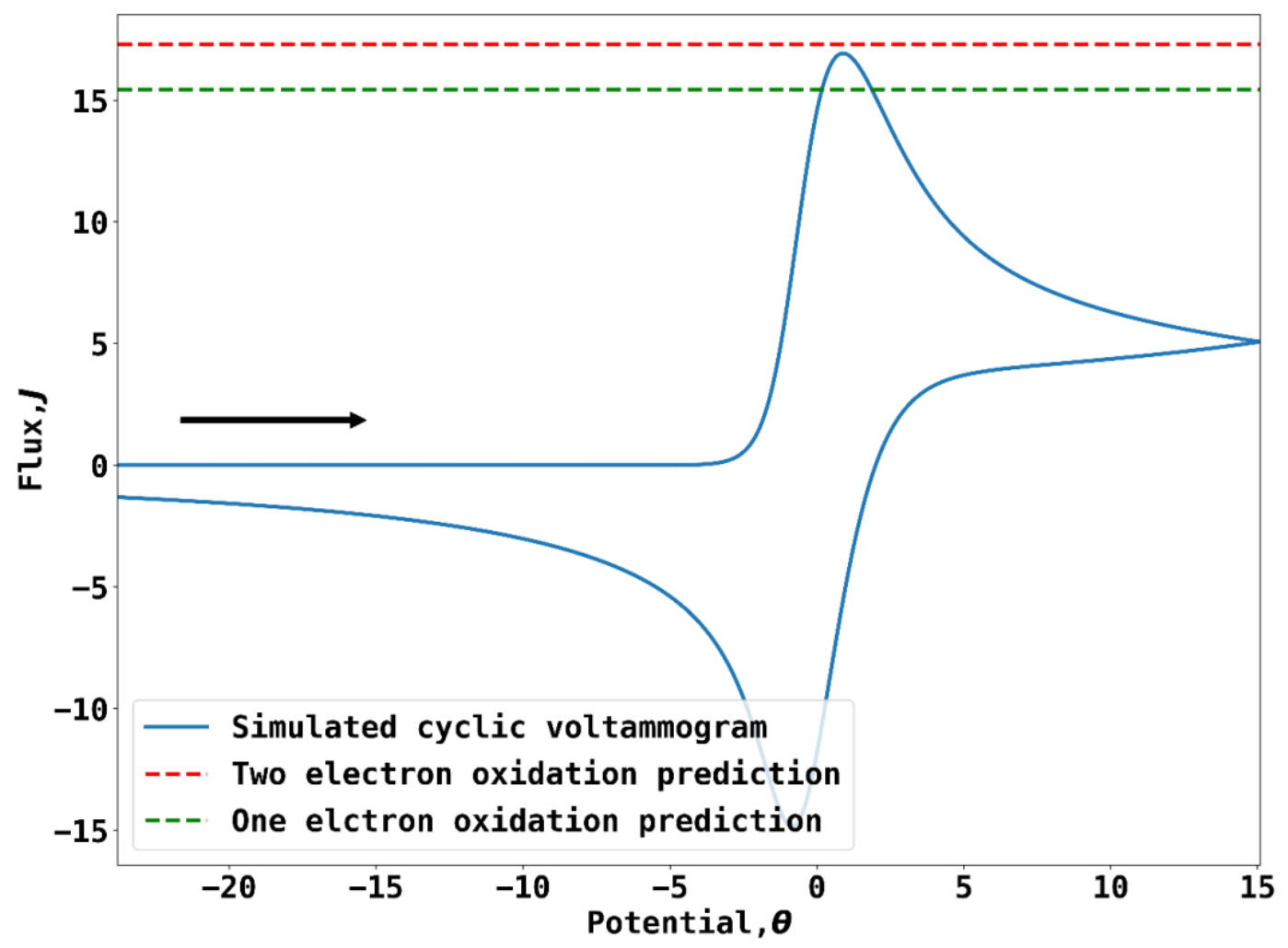

Fig. 8. Simulation of a cyclic voltammogram at a dimensionless scan rate of $\sigma=1198$, corresponding to a dimensional scan rate of $100 \mathrm{mV} / \mathrm{s}$ using the literature rate constants $k_{f}=1.5 \times 10^{9} \mathrm{M}^{-1} \mathrm{~s}^{-1}$ and $k_{b}=5 \times 10^{7} \mathrm{~s}^{-1}$. The blue line is the simulated cyclic voltammogram, the red dashed line is the predicted flux from Molina considering no follow up chemical reactions [3] and the green dashed line represents the predicted peak flux from ordinary one-electron oxidation Randles-Ševčik equation[1, 2]. The black arrow indicates the start and initial direction of potential sweep.

We next predict the response of cyclic voltammetry at different bulk concentration of bromide. When $\sigma=1198$, corresponding to dimensional scan rate of $100 \mathrm{mV} / \mathrm{s}, c_{B r^{-}}^{*}$ is varied from $10^{-5} \mathrm{M}$ to $10 \mathrm{M}$ with the following dimensional parameters $\left(\epsilon=0.8 \mathrm{~mm}, c_{B r_{2}}^{*}=0 M, c_{B r_{3}^{-}}^{*}=0 M, D_{B r^{-}}=2.08 \times\right.$ $\left.10^{-9} \mathrm{~m}^{2} / \mathrm{s}, D_{B r_{2}}=1.18 \times 10^{-9} \mathrm{~m}^{2} / \mathrm{s}, D_{B r_{3}^{-}}=1.145 \times 10^{-9} \mathrm{~m}^{2} / \mathrm{s}\right)$ and the results are shown in Fig. 9. When $c_{B r^{-}}^{*}$ is smaller than $0.1 M$, the voltammograms show a single peak corresponding to a simple two electron oxidation shown in Fig. 1, indicating that the cyclic voltammetry is under the control of the electrochemical reaction.

As shown in Fig. 9, increasing the bulk concentration of bromide above $0.1 \mathrm{M}$ facilitates the competing chemical reaction of forming tribromide with bromine and bromide, and thus decreasing the peak flux of the first peak. The second peak appears as tribromide decomposes to bromine and bromide, and bromide is then oxidized. Hence, based on the voltammograms shown in Fig. 9, at higher bulk concentration of bromide, usually higher than $1 M$, one can observe the first peak with a reduced height and the presence of the second peak. 


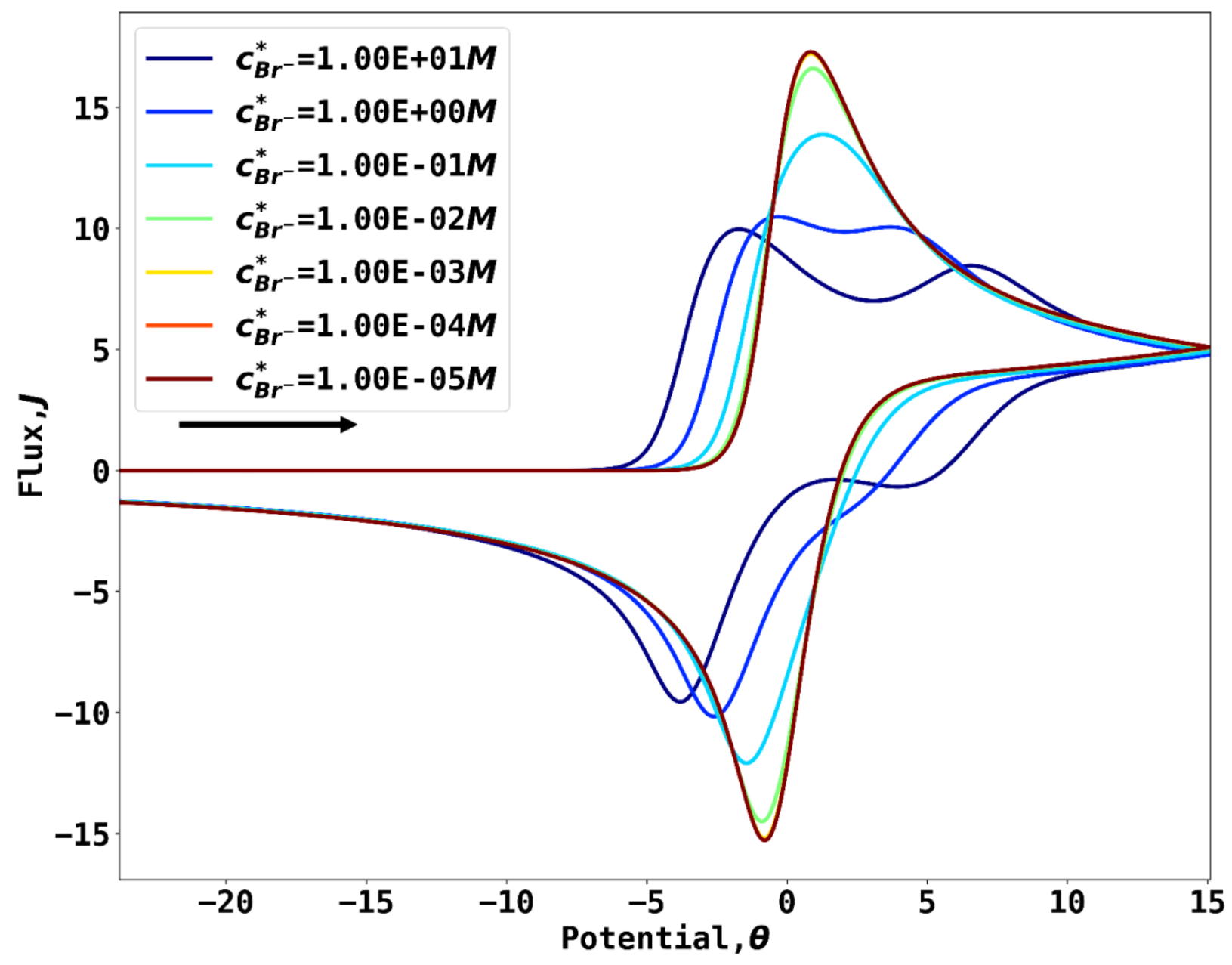

Fig. 9. Voltammetry showing the effects of varying the bulk concentration of bromide from $10^{-5} M$ to $10 \mathrm{M}$ at scan rate of $100 \mathrm{mV} / \mathrm{s}$. Second peak can be observed when $\mathrm{C}_{B r^{-}}^{*}$ is larger than $1 \mathrm{M}$. The black arrow indicates the start and initial direction of potential sweep.

To observe the effect of altering the scan rate on the bromine/bromide system, at two different bulk concentration of bromide $(5 \mathrm{mM}$ and $1 \mathrm{M})$, the scan rate is varied from $\sigma=0.012$ to $\sigma=1.2 \times 10^{5}$, corresponding to dimensional scan rate $10^{-6} \mathrm{~V} / \mathrm{s}$ to $10 \mathrm{~V} / \mathrm{s}$ with the dimensional parameters: $\left(\epsilon=0.8 \mathrm{~mm}, c_{B r_{2}}^{*}=0 M, c_{B r_{3}^{-}}^{*}=0 M, D_{B r^{-}}=2.08 \times 10^{-9} \mathrm{~m}^{2} / \mathrm{s}, D_{B r_{2}}=1.18 \times 10^{-9} \mathrm{~m}^{2} / \mathrm{s}, D_{B r_{3}^{-}}=\right.$ $\left.1.145 \times 10^{-9} \mathrm{~m}^{2} / \mathrm{s}\right)$. The chemical reaction rate constants $k_{f}$ and $k_{b}$ are the literature values mentioned above. In Fig. 10 and Fig. 11, when $c_{B r^{-}}^{*}=5 \mathrm{mM}$ and $1 M$ respectively, varying scan rate has no visible effect on the shape of the standardized voltammogram. Note that the dimensionless flux $J$, is standardized by dividing the flux with square root of dimensionless scan rate. 


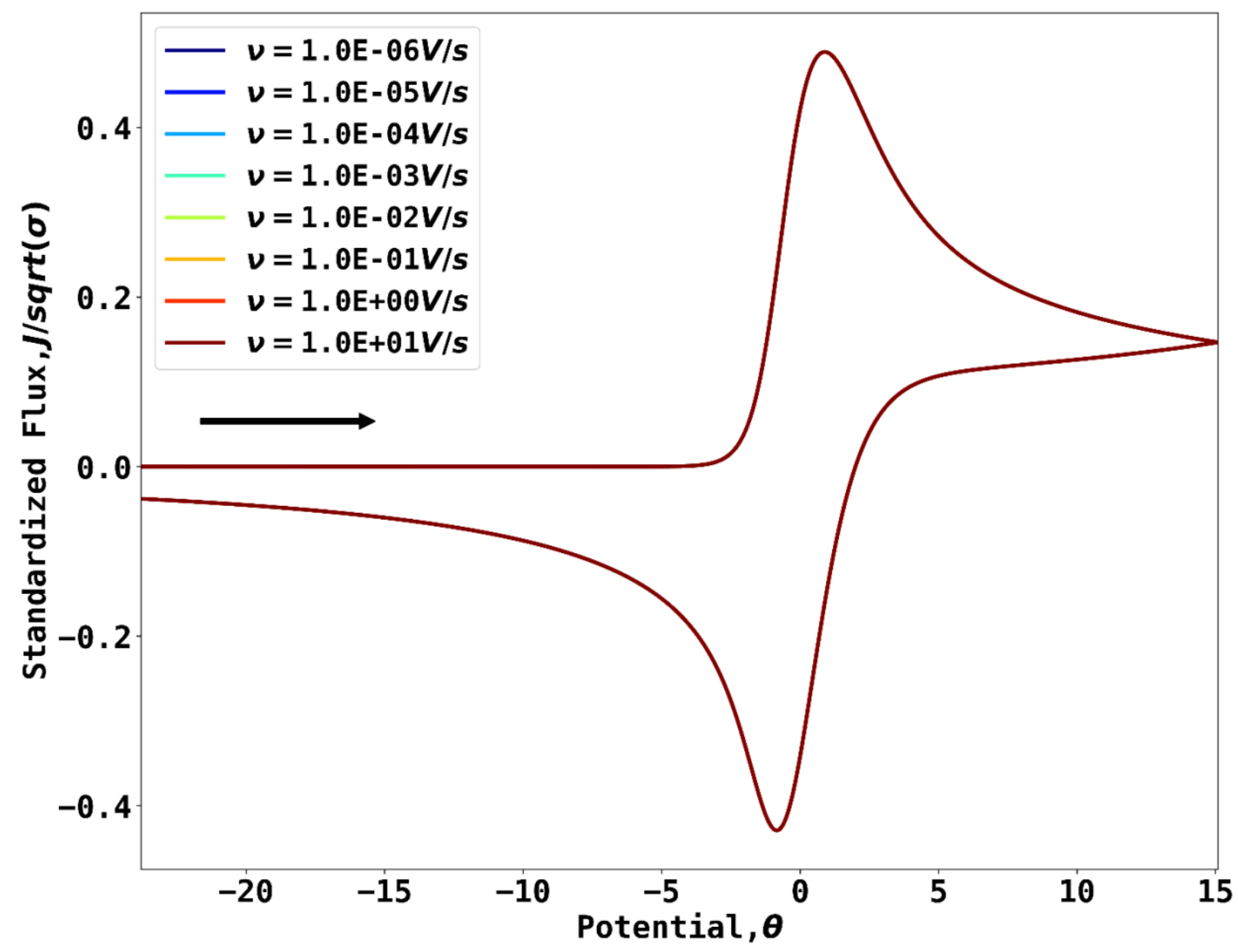

Fig. 10. Voltammograms simulated to show the effect of varying the scan rate from $10^{-6} \mathrm{~V} / \mathrm{s}$ to $10 \mathrm{~V} / \mathrm{s}$. When the bulk concentration of bromide is $5 \mathrm{mM}$ has no effect on the shape of standardized voltammogram: The second peak cannot be observed. Note that the flux is standardized against the square root of dimensionless scan rate. The black arrow indicates the start and initial direction of potential sweep. 


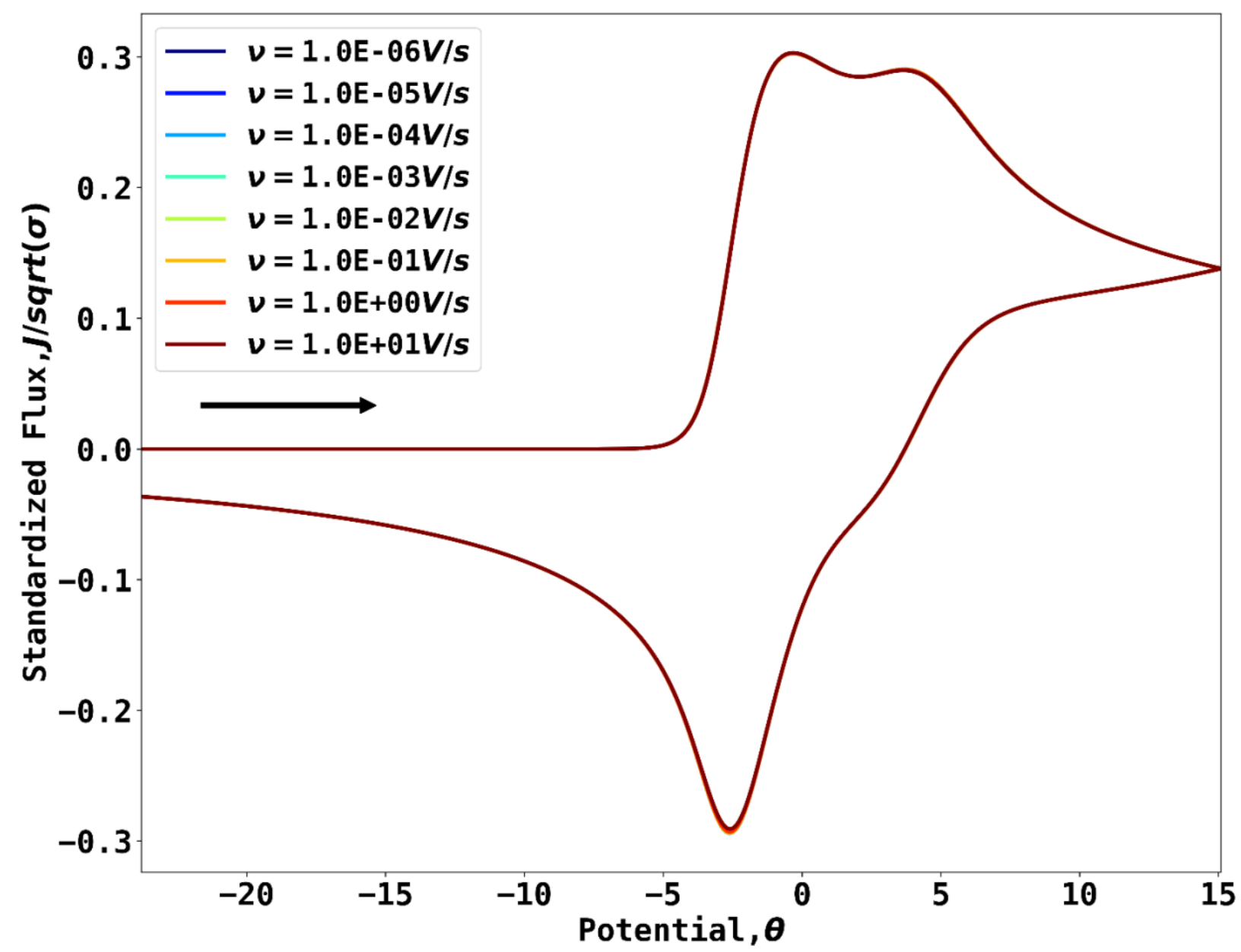

Fig. 11. Voltammetry showing that varying the scan rate from $10^{-6} \mathrm{~V} / \mathrm{s}$ to $10 \mathrm{~V} / \mathrm{s}$ when the bulk concentration of bromide is 1 $M$ has no effect on the shape of standardized voltammogram. The second peak can be observed in this plot. The black arrow indicates the start and initial direction of potential sweep.

\section{Experimental Methods}

The reaction electrolyte was prepared by dissolving $5.0 \mathrm{mM}$ of sodium bromide (May and Baker, UK) in $0.10 \mathrm{M} \mathrm{HNO}_{3}\left(\mathrm{HNO}_{3}>65 \%\right.$ assay, Fluka, UK). All solutions used ultrapure water of resistivity $18.2 \mathrm{M} \Omega \mathrm{cm}$ at $298 \mathrm{~K}$ (Millipore, USA).

Variable scan rate cyclic voltammetry was conducted using a three electrode system A platinum (Pt) electrode of diameter $1.5 \mathrm{~mm}$ and a glassy carbon (GC) electrode of diameter $3.0 \mathrm{~mm}$ were used after 
polishing with alumina slurries of progressively decreasing sizes: $1 \mu \mathrm{m}, 0.3 \mu \mathrm{m}, 0.05 \mu \mathrm{m}$. A saturated calomel electrode (SCE) served as the reference electrode and a graphite rod as the counter electrode.

Electrochemical experiments were performed using $\mu$ Autolab II potentiostat (Metrohm-Autolab BV, Utrecht, Netherlands). The computer software, GPES v.4.9 (Metrohm Autolab B.V., NL) was used to control and analyse all electrochemical experiments.

\section{Experimental Results and Discussion}

In the above, the voltammetry of the bromide oxidation in aqueous solution was predicted under conditions where the electron transfer was reversible and the follow up chemical reaction between bromide anions and bromine molecules leading to the formation of tribromide ions was characterised by the known equilibrium and rate constants for the reaction. In this section, we compare the predictions with experiment.

First steady-state voltammetry at a Pt microdisc electrode was used to measure the diffusion coefficient of the bromide ion in $0.1 \mathrm{M} \mathrm{HNO}_{3}$ (see $\mathrm{SI}$ ) yielding the value of $2.1 \times 10^{-9} \mathrm{~m}^{2} / \mathrm{s}$ which compares very well with the literature [29] value of $2.08 \times 10^{-9} \mathrm{~m}^{2} / \mathrm{s}$. The literature value was used in the simulations reported in the previous section.

Next cyclic voltammetry at a Pt macrodisc electrode (radius $0.75 \mathrm{~mm}$; geometric area $1.8 \times 10^{-2} \mathrm{~cm}^{2}$ ) was conducted on a similar solution. Fig. 12 shows the voltammetry of a $5.0 \mathrm{mM}$ solution of $\mathrm{NaBr}$ in $0.1 \mathrm{M} \mathrm{HNO}_{3}$ where the voltage scan range is from $0.5 \mathrm{~V}$ to $1.05 \mathrm{~V}$ (VS SCE) embracing the feature with a peak potential of $0.95 \mathrm{~V}$ (vS SCE) attributed to the reaction,

$$
2 \mathrm{Br}^{-}-2 e^{-} \rightleftarrows B r_{2}
$$

The inset to Fig. 12 shows that the peak current scales with the square root of the voltage scan rate as predicted. The linearity confirms a diffusion controlled electrode process. The slope of the line in the inset has a value of $1.23 \times 10^{-4} \frac{A}{\sqrt{V / S}}$ after a tiny $(<2 \%)$ baseline correction. This compares with a slope of $1.19 \times 10^{-4} \frac{A}{\sqrt{V / S}}$ predicted based on the simulations for the bromine/bromide system presented above and using literature parameters. Good agreement is apparent. Note that in contrast the expected slope, on the basis of the Randles-Ševčík equation for a simple one electron oxidation, $A-e^{-} \rightleftarrows B$, is $1.08 \times 10^{-4} \frac{A}{\sqrt{V / S}}$ whilst that for on the basis of Molina's equation [3] for $2 A-2 e^{-} \rightleftarrows B$ (but without any following chemical reaction is $1.21 \times 10^{-4} \frac{A}{\sqrt{V / S}}$. Note that the latter two calculated slopes assume the equality of the diffusion coefficients of bromide and bromine whereas the simulated slope uses the literature diffusion coefficients for each ion. It is evident that solution phase tribromide formation is negligible based on both the experimental and simulated data. Interestingly, however, Fig. 12b which shows the aqueous bromide oxidative voltammetry extended to more positive potentials, reveals a second wave with a peak potential of ca $1.2 \mathrm{~V}$ (Vs SCE). The origins of this second wave do not lie in the 
dissociation of tribromide ions exclusively in the solution phase on the voltammetric timescale. This conclusion assumes the accuracy of the literature data for the formation and dissociation rate constants for tribromide are in serious errors, since the above simulations and experiments show that negligible tribromide is formed at the bromide concentrations used in the present study. Indeed, simulations show that a concentration of ca $0.1 \mathrm{M}$ of bromide would be needed for sufficient tribromide ion to be generated for a second peak to be visible assuming all species are confined to the solution phase. At present the origins of the second feature are unclear but are likely to reside in the neglect of adsorbed species in the above model [32]. This is consistent with our observations that if platinum is replaced with glassy carbon as the electrode material no second peak is discernible except possibly at the very different potential of $+1.5 \mathrm{~V}$ (vS SCE) and obscured by solvent decomposition, an observation which hints at surface rather than solution chemistry operating.
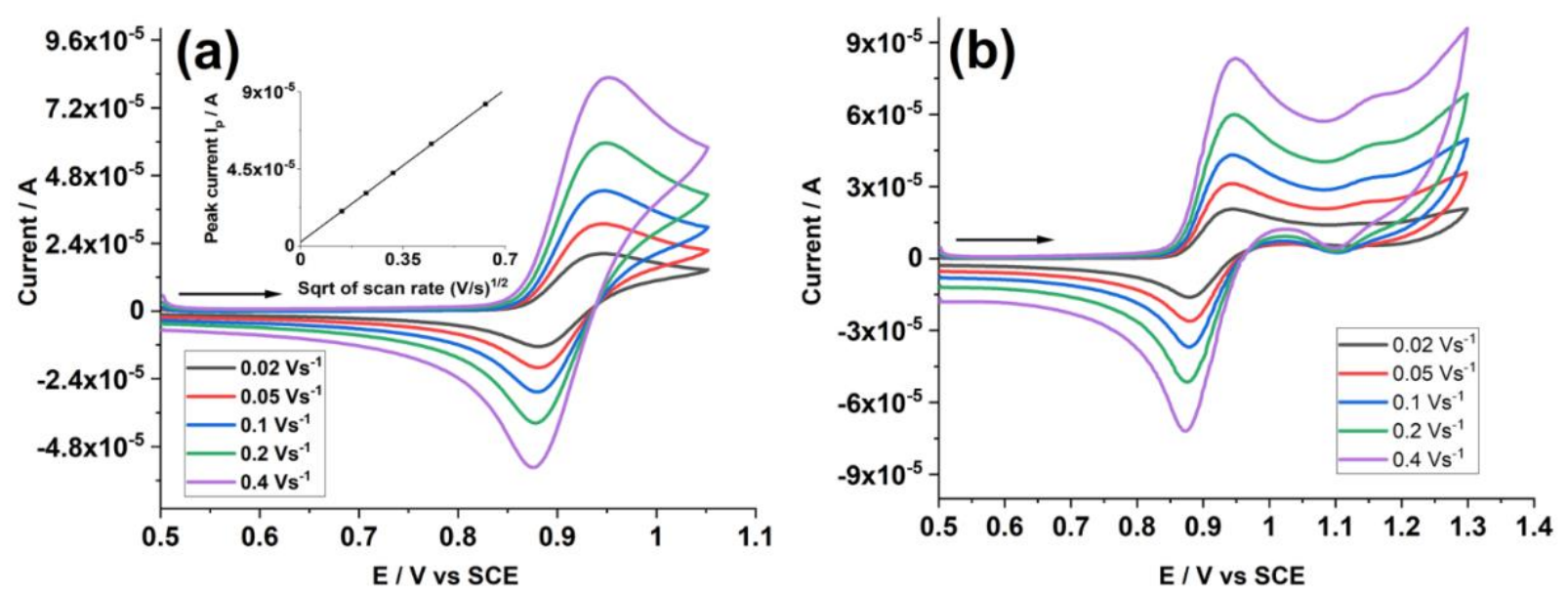

Fig. 12. Cyclic voltammograms of $5 \mathrm{mM} \mathrm{NaBr}$ in $0.1 \mathrm{mM} \mathrm{HNO}_{3}$ at Pt macro electrode of $\mathrm{r}=0.75 \mathrm{~mm}$ as a function of scan rates. a) voltage scan range from $0.5 \mathrm{~V}$ to $1.05 \mathrm{~V}$, inset; plot of oxidative peak current $\left(\mathrm{I}_{p}\right)$ vs square root of scan rate, $b$ ) voltage scan range from $0.5 \mathrm{~V}$ to $1.3 \mathrm{~V}$. The black arrow indicates the start and initial direction of potential sweep.

\section{Conclusions}

The reaction $2 A-2 e^{-} \rightleftarrows B$, of non-unity stoichiometry has been simulated under conditions of cyclic voltammetry at a macro-electrode. The effect of a follow up chemical reaction of the form $A+B \rightleftarrows C$ was specifically considered and shown, under certain critical conditions of concentration and scan rate, to give rise to two voltammetric peaks where the second results from the dissociation of $C$. The modelling was applied to the bromide oxidation system at platinum electrodes in aqueous solution with good quantitative agreement in respect of the first voltammetric wave, which is consistent with an electrochemically reversible oxidation forming molecular bromine, $B r_{2}$, with negligible tribromide formation. Further mechanistic work to refine this mechanism and to identify the role of adsorbed species, is on-going. 


\section{Acknowledgements}

Author Archana K S thanks the Commonwealth Scholarships Commissions UK and the University of Oxford for funding her DPhil research in the University of Oxford, UK.

\section{References}

[1] A. Ševčik, Oscillographic polarography with periodical triangular voltage, Collection of Czechoslovak Chemical Communications 13 (1948) 349-377.

[2] J.E.B. Randles, A cathode ray polarograph. Part II.-The current-voltage curves, Transactions of the Faraday Society 44(0) (1948) 327-338.

[3] J.M. Gomez-Gil, E. Laborda, A. Molina, General Explicit Mathematical Solution for the Voltammetry of Nonunity Stoichiometry Electrode Reactions: Diagnosis Criteria in Cyclic Voltammetry, Anal Chem 92(5) (2020) 3728-3734.

[4] K. Cathro, K. Cedzynska, D. Constable, P. Hoobin, Selection of quaternary ammonium bromides for use in zinc/bromine cells, Journal of power Sources 18(4) (1986) 349-370.

[5] D.J. Eustace, Bromine complexation in zinc - bromine circulating batteries, Journal of the Electrochemical Society 127(3) (1980) 528.

[6] S.J. Yoo, B. Evanko, X. Wang, M. Romelczyk, A. Taylor, X. Ji, S.W. Boettcher, G.D. Stucky, Fundamentally addressing bromine storage through reversible solid-state confinement in porous carbon electrodes: design of a high-performance dual-redox electrochemical capacitor, Journal of the American Chemical Society 139(29) (2017) 9985-9993.

[7] Q. Li, M. Haque, V. Kuzmenko, N. Ramani, P. Lundgren, A.D. Smith, P. Enoksson, Redox enhanced energy storage in an aqueous high-voltage electrochemical capacitor with a potassium bromide electrolyte, Journal of Power Sources 348 (2017) 219-228.

[8] K.S. Archana, R. pandiyan Naresh, H. Enale, V. Rajendran, A.V. Mohan, A. Bhaskar, P. Ragupathy, D. Dixon, Effect of positive electrode modification on the performance of zinc-bromine redox flow batteries, Journal of Energy Storage 29 (2020) 101462.

[9] F. Yu, L. Pang, X. Wang, E.R. Waclawik, F. Wang, K.K. Ostrikov, H. Wang, Aqueous alkaline-acid hybrid electrolyte for zinc-bromine battery with 3V voltage window, Energy Storage Materials 19 (2019) 56-61.

[10] O.W. Kolling, The Bromine-Tribromide Ion Equilibrium in Anhydrous Acetic Acid, Transactions of the Kansas Academy of Science (1903-) 73(4) (1970) 452-459. 
[11] G.D. Allen, M.C. Buzzeo, C. Villagrán, C. Hardacre, R.G. Compton, A mechanistic study of the electrooxidation of bromide in acetonitrile and the room temperature ionic liquid, 1-butyl-3-methylimidazolium bis(trifluoromethylsulfonyl)imide at platinum electrodes, Journal of Electroanalytical Chemistry 575(2) (2005) 311-320.

[12] R. Caminiti, M. Carbone, G. Mancini, C. Sadun, Study of cetyltrialkylammonium bromide and tribromide salts in thesolid phase, Journal of Materials Chemistry 7(8) (1997) 1331-1337.

[13] T. Iwasita, M.C. Giordano, Kinetics of the bromine-tribromide-bromide redox processes on platinum electrodes in acetonitrile solutions, Electrochimica Acta 14(10) (1969) 1045-1059.

[14] R.O. Griffith, A. McKeown, A.G. Winn, The bromine-bromide-tribromide equilibrium, Transactions of the Faraday Society 28 (1932) 101-107.

[15] M.F. Ruasse, J. Aubard, B. Galland, A. Adenier, Kinetic study of the fast halogen-trihalide ion equilibria in protic media by the Raman-laser temperature-jump technique. A non-diffusion-controlled ion-molecule reaction, The Journal of Physical Chemistry 90(18) (1986) 4382-4388.

[16] R.G. Compton, C.E. Banks, Understanding voltammetry, 3rd ed., World Scientific 2018.

[17] R.G. Compton, E. Laborda, E. Kaetelhoen, Understanding voltammetry: simulation of electrode processes, World Scientific 2020.

[18] J.K. Novev, R.G. Compton, Natural convection effects in electrochemical systems, Current Opinion in Electrochemistry 7 (2018) 118-129.

[19] J.A.V. Butler, Studies in heterogeneous equilibria. Part II. - The kinetic interpretation of the nernst theory of electromotive force, Transactions of the Faraday Society 19(March) (1924) 729-733.

[20] T. Erdey-Grúz, M. Volmer, Zur theorie der wasserstoff überspannung, Zeitschrift für physikalische Chemie 150(1) (1930) 203-213.

[21] A. Fick, Ueber Diffusion, Annalen der Physik 170(1) (1855) 59-86.

[22] A. Fick, V. On liquid diffusion, The London, Edinburgh, and Dublin Philosophical Magazine and Journal of Science 10(63) (1855) 30-39.

[23] A. Einstein, On the motion of small particles suspended in liquids at rest required by the molecularkinetic theory of heat, J Annalen der physik 17 (1905) 549-560.

[24] I.B. Svir, A.I. Oleinick, R.G. Compton, Solution of Ring Electrode Problems in Spherical Coordinates: An Application to Near-Steady-State Linear Sweep Voltammetry, Russian Journal of Electrochemistry 39(2) (2003) 160-164.

[25] E. Kätelhön, R.G. Compton, Testing and validating electroanalytical simulations, Analyst 140(8) (2015) 2592-2598. 
[26] E. Kätelhön, R.G. Compton, Correction: Testing and validating electroanalytical simulations, Analyst 141(3) (2016) 1154-1154.

[27] E. Kätelhön, R.G. Compton, Correction: Testing and validating electroanalytical simulations, Analyst 140(9) (2015) 3290-3290.

[28] P. Adanuvor, R.E. White, S. Lorimer, The effect of the tribromide complex reaction on the oxidation/reduction current of the $\mathrm{Br} 2 / \mathrm{Br}$ - electrode, Journal of The Electrochemical Society 134(6) (1987) 1450.

[29] R.E. White, S. Lorimer, A model of the bromine/bromide electrode reaction at a rotating disk electrode, Journal of the Electrochemical Society 130(5) (1983) 1096.

[30] W.M. Haynes, CRC handbook of chemistry and physics, CRC press2014.

[31] E.L. Cussler, E.L. Cussler, Diffusion: mass transfer in fluid systems, Cambridge university press2009.

[32] J. Xu, N.S. Georgescu, D.A. Scherson, The oxidation of bromide on platinum electrodes in aqueous acidic solutions: electrochemical and in situ spectroscopic studies, Journal of The Electrochemical Society 161(6) (2014) H392. 